\title{
AVALIAÇÃO DOS PARÂMETROS FISIOLÓGICOS EM RECÉM NASCIDOS PRÉ-TERMO EM VENTILAÇÃO MECÂNICA APÓS PROCEDIMENTOS DE FISIOTERAPIA NEONATAL.
}

\author{
EVALUATION OF THE PHYSIOLOGICAL PARAMETERS IN PRE-TERM \\ NEWBORN IN MECHANICAL VENTILATION AFTER PROCEDURES OF \\ NEONATAL PHYSIOTHERAPY.
}

\author{
Cláudia de Castro Selestrin ${ }^{*+}$ \\ Adriana Gonçalves de Oliveira* \\ Celso Ferreira ** \\ Arnaldo Augusto Franco de Siqueira*** \\ Luiz Carlos de Abreu* \\ Neif Murad*
}

Selestrin CC, Oliveira AG, Ferreira C, Siqueira AAF, Abreu LC, Murad N. Avaliação dos parâmetros fisiológicos em recém nascidos pré-termo em ventilação mecânica após procedimentos de fisioterapia neonatal. Rev Bras Crescimento Desenvolv Hum 2007; 17(1):146-155.

Resumo: A fisioterapia nas Unidades de Cuidados Intensivos Neonatais pode ser considerada nova modalidade de terapia. Acredita-se que o acompanhamento fisioterapêutico dos recémnascidos seja capaz de proporcionar uma estabilidade da freqüência cardíaca, da pressão arterial, freqüência respiratória e saturação de oxigênio, bem como preserva a temperatura corporal, promovendo a manutenção funcional da circulação cerebral do recém-nascido e secundariamente, mantém as vias aéreas com fluxo menos turbulento possível e com o mínimo de secreção, permitindo um aumento na permeabilidade e redução do número de fatores intrínsecos das vias aéreas que contribuem para o aumento da resistência pulmonar e diminuição nos eventos fisiológicos de trocas gasosas. Outros autores apresentam efeitos deletérios da fisioterapia sobre a população neonatal. Estas controvérsias em relação à fisioterapia respiratória neonatal nos induziram a estudar os efeitos de tal procedimento sobre os parâmetros fisiológicos. O objetivo foi analisar os efeitos da prática de fisioterapia neonatal sobre os parâmetros fisiológicos frequiência cardíaca, frequiência respiratória, saturação de oxigênio, pressão arterial e temperatura axilar em recémnascidos pré-termo submetidos à ventilação mecânica. Realizou-se um estudo prospectivo, na UTI neonatal do Hospital Municipal Universitário de São Bernardo do Campo, no período de janeiro a dezembro de 2005. Foi feita a análise descritiva das variáveis do estudo. Para comparar se os valores médios antes e depois da fisioterapia são estatisticamente diferentes foi realizado o teste Anova de medidas repetidas. E em seguida foi realizado o teste de comparações múltiplas de Newman-Keuls para as variáveis freqüência cardíaca, frequiência respiratória, saturação de oxigênio, e o teste-t pareado para as variáveis pressão arterial e temperatura axilar. O nível de significância considerado foi de 5\%. A amostra foi constituída de 27 recém-nascidos pré-termo, que permaneceram sob ventilação mecânica invasiva e procedimentos intervencionistas de fisioterapia. Observou-se uma redução da frequiência cardíaca, frequiência respiratória, ao longo das sessões clínicas de fisioterapia neonatal, sem alteração da pressão arterial, aumento da saturação de hemoglobina pelo oxigênio e diminuição da temperatura porém sem repercussões clínicas. Vários fatores podem influenciar na estabilidade dos parâmetros fisiológicos, bem como na

Departamento de Fisiologia da Faculdade de Medicina do ABC - FMABC

Serviço de Fisioterapia do Hospital Municipal Universitário de São Bernardo do Campo da Faculdade de Medicina do ABC - HMU / FMABC.

** Professor Adjunto da Disciplina de Cardiologia da Universidade Federal de São Paulo - UNIFESP.

**** Professor Titular de Saúde Materno-Infantil da Faculdade de Saúde Pública da Universidade de São Paulo. 
monitorização de cada um deles. Dentre eles, as características da fisiologia neonatal, os fatores ambientais e o tipo de intervenção que é realizada, ou seja, a fisioterapia adaptada à situação de cada recém-nascido, irá determinar os possíveis resultados encontrados sobre os parâmetros fisiológicos dos RNPT. Em conclusão, a fisioterapia neonatal demonstrou ser um procedimento terapêutico sem repercussões deletérias em relação à variação dos parâmetros fisiológicos para o tratamento da população estudada.

Palavras-chave: Estabilidade hemodinâmica. Fisioterapia neonatal. Parâmetros fisiológicos. Recém-nascido.

\section{INTRODUÇÃO}

A fisioterapia neonatal consiste em procedimentos realizados pelo fisioterapeuta no período situado entre o clampeamento do cordão umbilical até 28 dias após o parto, que compreendem o manuseio da parte motora e pulmonar do recémnascido $(\mathrm{RN})^{1-3}$. Um dos objetivos do manuseio pulmonar é a remoção das secreções brônquicas em excesso. Os efeitos prejudiciais originados das secreções em excesso e o fato de que sua remoção pode, significantemente, melhorar a condutância específica das vias aéreas foi demonstrado em algumas revisões sistemáticas ${ }^{4,5}$.

O acompanhamento fisioterapêutico destes RNs proporciona uma estabilidade de variáveis hemodinâmicas, tal qual a frequiência cardíaca (FC), a manutenção funcional da circulação cerebral do RN e secundariamente, mantém as vias aéreas com fluxo menos turbulento possível e com o mínimo de secreção, permitindo um aumento na permeabilidade e redução do número de fatores intrínsecos das vias aéreas que contribuem para o aumento da resistência pulmonar e diminuição nos eventos fisiológicos de trocas gasosas ${ }^{1,3,6,7}$.

Há controvérsias referentes à fisioterapia respiratória no período neonatal. Estudos clínicos demonstraram benefícios da fisioterapia em recém-nascidos pré-termo (RNPT) através da melhora da mecânica pulmonar. Esses estudos tiveram como foco principal a função pulmonar. Noutros estudos observou-se a redução da variabilidade hemodinâmica desses RNPT, sendo destacado com efeitos terapêuticos benéficos dos procedimentos intervencionistas de fisioterapia neonatal (PIFN) $)^{3,8,9,16,24,25}$.

Entretanto, estudos realizados na década de 90 do século passado relatam efeitos deletérios, sugerindo que o manuseio por procedimentos intervencionistas de fisioterapia em RNs resulta em instabilidade hemodinâmica, sendo, portanto contra-indicados ${ }^{17,18}$. Nos trabalhos de Antunes et $\mathrm{al}^{8}$ e Silva et al ${ }^{19}$ realizou-se comparações do impacto de diversas técnicas de fisioterapia sobre os parâmetros fisiológicos. Esses autores concluíram que os resultados são divergentes e que novos estudos são necessários nessa população amplamente assistida na Unidade de Cuidados Neonatais.

Dessa maneira, em virtude dessas controvérsias em relação à fisioterapia neonatal como PIFN na população de RNPT, o objetivo deste estudo consiste em analisar a variabilidade dos parâmetros FC e frequiência respiratória (FR), saturação de hemoglobina pelo oxigênio $\left(\mathrm{SpO}_{2}\right)$, pressão arterial (PA) e temperatura (T) em RNPT e em ventilação mecânica, antes e após os PIFN.

\section{MÉTODO}

Realizou-se um estudo clínico prospectivo de caráter experimental intervencionista. A população em estudo constituiu-se de 27 recém-nascidos pré-termo sob ventilação mecânica invasiva, na Unidade de Terapia Intensiva Neonatal do Hospital Municipal Universitário de São Bernardo do Campo da Faculdade de Medicina do ABC.

O estudo foi realizado de janeiro a dezembro de 2005, após aprovação do protocolo pelo Comitê de Ética da Faculdade de Medicina do ABC (n $\left.{ }^{\circ} 146 / 2004\right)$ e após a assinatura do termo de consentimento livre e esclarecido por parte de um dos responsáveis pelo recém-nascido.

Optou-se pela não utilização de grupo controle, pois cada RN foi parâmetro de si mesmo, através da comparação dos momentos pré e pósfisioterapia. 
Os critérios de inclusão nessa pesquisa clínica foram: peso ao nascer $\geq 500$ gramas, indicação clínica para procedimentos intervencionistas com prescrição dos procedimentos fisioterapêuticos, bem como estar em uso de ventilação pulmonar mecânica através de cânula traqueal, ter idade gestacional igual ou inferior a 36 semanas avaliada pelo método de Capurro $^{20} \mathrm{e}$ ausência de instabilidade hemodinâmica, mal-formações congênitas ou diagnóstico ultrassonográfico de hemorragia intracraniana.

Foram considerados como critérios de exclusão os RNPT que se encontravam em uso de analgesia e/ou sedação no momento da aplicação do protocolo.

As variáveis $\mathrm{FC}, \mathrm{SpO}_{2}$ e PA foram mensuradas e compiladas através de um monitor multiparamétrico da marca Dixtal, modelo DX2020.

A variável FR foi mensurada com auxilio de um cronômetro da marca Casio modelo FS02 seguida de quantificação, sempre pelo mesmo pesquisador e em 60 segundos, através dos movimentos tóraco-abdominais.

A variável temperatura foi aferida durante 5 minutos através de um termômetro de mercúrio na região axilar.

Os RNPT foram ventilados em ventilador mecânico da marca Newport, modelo NMI na modalidade Ventilação Mandatória Intermitente (IMV). Os parâmetros ventilatórios foram ajustados de acordo com a necessidade de cada RNPT. Nenhum ajuste dos parâmetros foi realizado durante a aplicação do protocolo.

O protocolo obedeceu à seguinte seqüência: monitorização, fisioterapia motora, fisioterapia respiratória, fisioterapia motora e monitorização. Esta seqüência de procedimentos foi denominada de procedimento intervencionista de fisioterapia neonatal (PIFN), sendo que a monitorização dos RNs foi feita antes e após os PIFN, onde o préPIFN referiu-se a monitorização antes da fisioterapia neonatal e o pós-PIFN após a fisioterapia neonatal, de acordo com Abreu et $\mathrm{al}^{3}$.

Os dados foram obtidos a partir de única sessão clínica de fisioterapia, realizada entre 7 e 8 horas da manhã, sendo compilados durante 09 minutos antes e mais 09 minutos imediatamente após o término dos PIFN. Os dados foram coletados pelo fisioterapeuta pesquisador e os PIFN foram realizados por um fisioterapeuta assistencial da Unidade de Cuidados Intensivos Neonatais que não tinha acesso às fichas de coleta de dados.

As variáveis $\mathrm{FC}$ e $\mathrm{SpO}_{2}$ foram compiladas no monitor multiparamétrico que captava um registro por segundo e o valor médio foi calculado a cada 180 segundos através de média aritmética. Portanto, para cada um dos períodos 0 a 3 minutos, 3 a 6 minutos e 6 a 9 minutos um valor médio foi obtido, denominados como PIFN3, PIFN6 e PIFN9 respectivamente. Adotou-se procedimento idêntico para a variável FR, porém os dados foram listados minuto a minuto pelo pesquisador e as médias para cada período foram calculadas posteriormente. Este procedimento foi realizado no período pré-PIFN e pós-PIFN.

A variável temperatura axilar foi mensurada a partir do primeiro ao quinto minuto da avaliação pré-PIFN, constituindo-se da medida pré-PIFN, e a segunda pós-PIFN foi realizada do quarto ao nono minuto pós-PIFN.

A variável pressão arterial foi mensurada no primeiro minuto pré-PIFN e ao final do nono minuto pós-PIFN.

Procedeu-se à aspiração da cânula orotraqueal por sistema aberto. A fração inspirada de oxigênio ( $\mathrm{FiO}_{2}$ do ventilador mecânico) estabelecida pela equipe médica de acordo com a gasometria arterial, permaneceu constante durante todo a sessão clínica de fisioterapia neonatal, haja vista que os RNs mantiveram-se sendo avaliados ininterruptamente do ponto de vista clínico e apresentaram boa perfusão periférica e central durante todo o procedimento protocolar. Em nenhum caso dos 27 RNs estudados houve necessidade de aumento da fração inspirada de oxigênio $\left(\mathrm{FiO}_{2} \%\right)$, de maneira que a equipe assistencial não procedeu a mudanças nesta variável, mesmo após os PIFN. Assim, foram respeitados os preceitos éticos relacionados à soberania da clínica em qualquer procedimento realizado na condução de pacientes criticamente enfermos e em ambiente de cuidados intensivos.

Todos os RNs permaneceram na posição supina por pelo menos 30 minutos antes da fisioterapia até o final da coleta de dados. 


\section{Análise estatística}

Procedeu-se a análise descritiva das variáveis qualitativas que foram apresentadas em termos de seus valores absolutos e relativos. Quanto às variáveis quantitativas, essas foram apresentadas através de média e desvio padrão.

Para comparar se os valores médios antes e depois da fisioterapia são estatisticamente diferentes foi realizado o teste Anova de medidas repetidas (oneway). Em seguida foi realizado o teste de comparações múltiplas de Newman-Keuls para as variáveis: freqüência cardíaca, freqüência respiratória, saturação de oxigênio e o teste " $t$ " de Student para as variáveis: pressão arterial e temperatura axilar.

Em todos os testes fixou-se em 5\% ( $p<0,05)$ o nível para rejeição da hipótese de nulidade. Foram utilizados os softwares específicos para estudos estatísticos, o SPSS 13.0 for windows e o Stata.

\section{RESULTADOS}

Dos 27 RNS estudados, $62,9 \%$ eram do sexo feminino, o peso médio e a idade gestacional em dias de gestação, obtida através do ultra-som (US) e pelo método Capurro, são apresentados na Tabela 1.

O tempo médio de duração do procedimento intervencionista de fisioterapia neonatal (PIFN) foi de 8 minutos e 53 segundos, com tempo mínino de 6 minutos e 13 segundos e máximo de 11 minutos e 06 segundos.

Os resultados médios e desvio padrão (DP) dos momentos extraídos das variáveis FC, FR e $\mathrm{SpO}_{2}$ estão apresentados na Tabela 2.

Na variável FC houve uma diferença estatisticamente significante quando comparados os valores médios pré-PIFN e pós-PIFN ( $\mathrm{p}=0,003$ ). Quando se compara em períodos, observou-se que houve diferença estatística significante em duas situações, entre os períodos pré-PIFN9e o período pós-PIFN9 ( $\mathrm{p}=0,007)$ e entre os períodos pós-PIFN3 e o período pós-PIFN9 ( $\mathrm{p}=0,001)$ evidenciando uma diminuição significativa (tabela 3 e gráfico 1 ) do primeiro período analisado em relação ao último. Noutra análise, observou-se uma diminuição estatisticamente significante na comparação da primeira medida após o final do PIFN em relação à última medida, sugerindo um período de estabilização.
Sobre a variável freqüência respiratória (FR), observou-se uma diferença estatisticamente significante quando comparados os valores médios préPIFN e pós-PIFN ( $\mathrm{p}=0,001)$. E quando comparados cada um dos períodos avaliados, observou-se diferença estatística significante em algumas situações, entre os períodos pré-PIFN9e o período pósPIFN9 ( $p<0,001)$, entre os períodos pré-PIFN9 e o período pós-PIFN6 ( $\mathrm{p}=0,001)$, entre os períodos pré-PIFN6 e o período pós-PIFN3 ( $\mathrm{p}=0,02)$, entre os períodos pré-PIFN6 e o período pós-PIFN6 ( $\mathrm{p}<0,001)$, entre os períodos pré-PIFN6 e o período pós-PIFN9 $(\mathrm{p}<0,001)$, entre os períodos préPIFN3 e o período pós-PIFN6 ( $p<0,001)$, entre os períodos pré-PIFN3 e operíodo pós-PIFN9 ( $p<0,001$ ), entre os períodos pós-PIFN3 e o período pósPIFN9 ( $p<0,008$ ), demonstrando que houve uma diminuição significativa como pode ser observado na tabela 2 e gráfico 2 , do primeiro período analisado em relação ao último, e também a diminuição foi significativa quando se comparou a primeira medida após o final do PIFN em relação à última medida, demonstrando um período de estabilização idêntico ao da FC.

Em relação à variável saturação de oxigênio $\left(\mathrm{SpO}_{2}\right)$, observou-se que houve uma diferença estatisticamente significante quando comparados os valores médios pré-PIFN e pós-PIFN ( $\mathrm{p}=0,003)$. E quando comparados cada um dos períodos avaliados, observou-se diferença estatística significante em cinco situações, entre os períodos pré-PIFN9 e o período pós-PIFN9 $(\mathrm{p}=0,026)$ entre os períodos pré-PIFN6 e o período pósPIFN9 ( $\mathrm{p}=0,021)$, entre os períodos pré-PIFN3 e o pós-PIFN6 $(\mathrm{p}=0,036)$ e entre os períodos pré-PIFN3 e o pós-PIFN9 ( $\mathrm{p}=0,014)$, demonstrando que houve um aumento significativo como pode ser observado na tabela 2 e gráfico 3 , do primeiro período analisado em relação ao último.

A pressão arterial sistêmica foi aferida no primeiro e último minuto de aplicação do protocolo. Os resultados obtidos através do teste Anova, da pressão sistólica e diastólica não mostraram diferença significativa $(\mathrm{p}=0,23)$, conforme destacado na tabela 3 e no gráfico 4 .

Em relação à temperatura, comparandose os resultados dos valores pré-PIFN e pósPIFN obteve-se queda estatisticamente significante $(\mathrm{p}=0,001)$. 
Tabela 1. Medidas-resumo das médias das variáveis peso ao nascimento, idade gestacional pelo exame ultrassonográfico (IG US) e idade gestacional avaliada pelo método Capurro.

\begin{tabular}{lccc}
\hline & Mínima & Média (DP) & Máxima \\
\hline Peso nascimento (gr) & 590 & $1043(+/-352)$ & 1740 \\
\hline IG US (dias) & 175 & $190,22(+/-36)$ & 224 \\
\hline IG Capurro (dias) & 175 & $200,88(+/-17)$ & 230 \\
\hline
\end{tabular}

DP: desvio padrão

Tabela 2. Medidas resumo das médias das variáveis freqüência cardíaca, frequiência respiratória, e saturação de oxigênio em 27 RNPT aferidas antes (pré-PIFN) da sessão clínica de fisioterapia e após (pós-PIFN) o término da sessão

\begin{tabular}{lcccccc}
\hline & Pré-PIFN3 & Pré-PIFN6 & Pré-PIFN9 & Pós-PIFN3 & Pós-PIFN6 & Pós-PIFN9 \\
\hline & Média (DP) & Média (DP) & Média (DP) & Média (DP) & Média (DP) & Média (DP) \\
\hline FC & $147,1(+/-21,14)$ & $143,1(+/-13,51)$ & $146,2(+/-14,94)$ & $148,2(+/-15,04)$ & $143,1(+/-12,78)$ & $139,5(+/-11,08)$ \\
\hline FR & $41,6(+/-5,91)$ & $42,3(+/-5,56)$ & $41,9(+/-5,85)$ & $40,3(+/-5,79)$ & $39,0(+/-5,50)$ & $38,2(+/-5,64)$ \\
\hline SpO $_{2}$ & $96,7(+/-1,74)$ & $96,6(+/-2,16)$ & $96,6(+/-2,23)$ & $97,1(+/-1,48)$ & $97,5(+/-1,34)$ & $97,6(+/-1,41)$ \\
\hline
\end{tabular}

DP: desvio padrão

Tabela 3. Medidas resumo das médias das variáveis pressão sistólica e pressão diastólica em 27 RNPT aferidas antes (pré) da sessão clínica de fisioterapia e após(pós) o término da sessão.

\begin{tabular}{cccc}
\hline Período & N & Média & Desvio Padrão \\
\hline PS Pré-PIFN & 27 & 61,3 & 4,62 \\
\hline PS Pós-PIFN & 27 & 61,5 & 3,27 \\
\hline PDPré-PIFN & 27 & 30,8 & 6,84 \\
\hline PDPós-PIFN & 27 & 30,8 & 6,35 \\
\hline
\end{tabular}

Tabela 4. Medidas-resumo das médias da variável temperatura axilar em 27 RNPT aferidas antes (pré) da sessão clínica de fisioterapia e após (pós) o término da sessão.

\begin{tabular}{cccc}
\hline Período & N & Média & Desvio Padrão \\
\hline Pré-PIFN & 27 & 36,5 & 0,40 \\
\hline Pós-PIFN & 27 & 36,3 & 0,30 \\
\hline
\end{tabular}

Gráfico 1. Variação média de frequiência cardíaca nos 6 períodos avaliados.

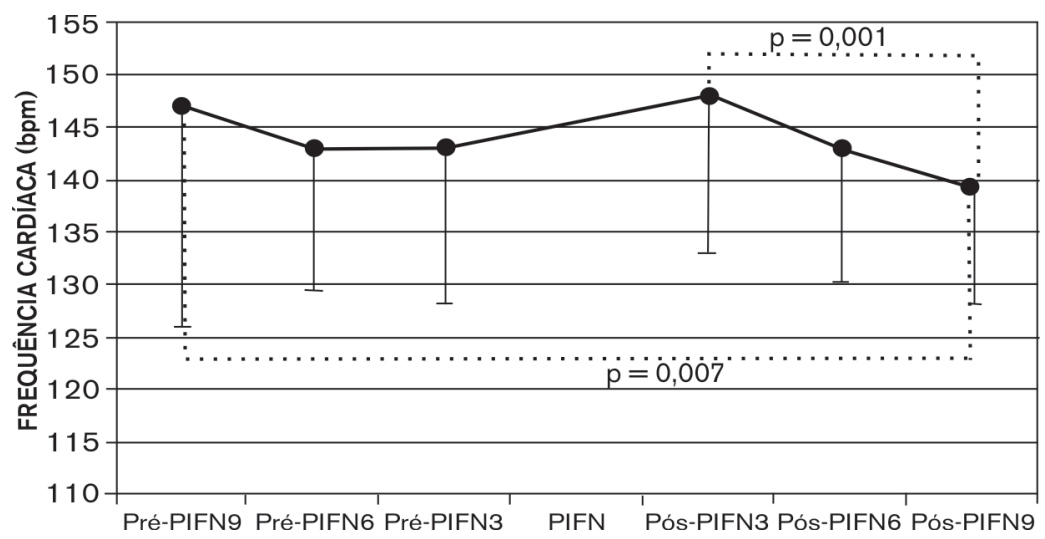


Gráfico 2. Variação média de freqüência respiratória nos 6 períodos avaliados.

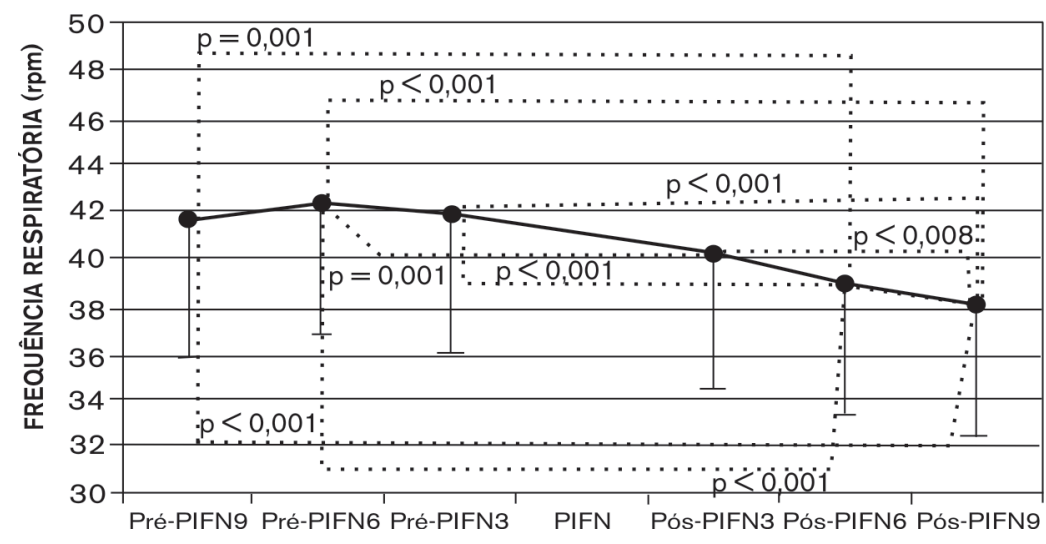

Gráfico 3. Variação média da saturação de oxigênio nos 6 períodos avaliados.

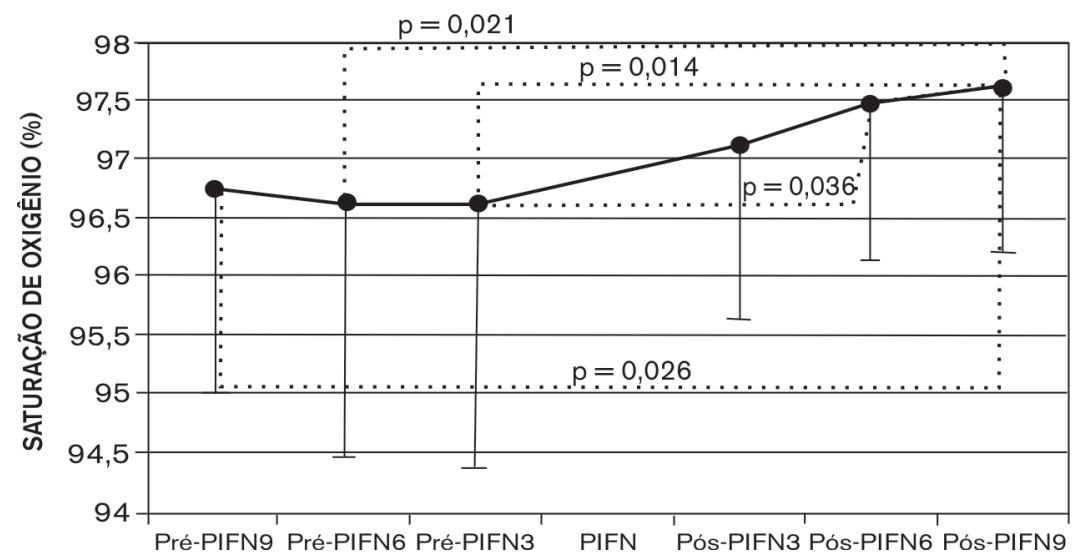

Gráfico 4. Variação média da pressão arterial antes e após os PIFN.

Gráfico 5. Variação média da temperatura axilar antes e após os PIFN.
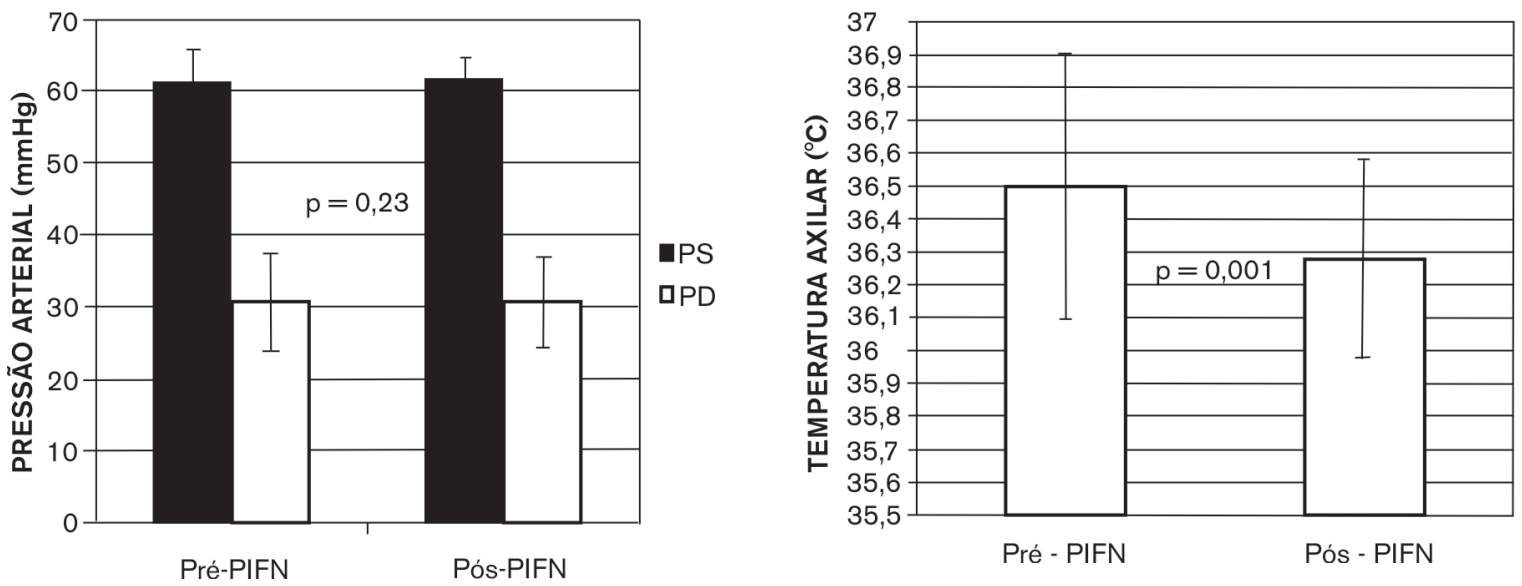


\section{DISCUSSÃO}

Avaliando-se o conjunto das variáveis fisiológicas analisadas encontrou-se diminuição da FC, FR e T, aumento da $\mathrm{SpO}_{2} \%$ e manutenção da PA.

Respostas diferentes em relação aos parâmetros fisiológicos podem ocorrer em níveis de desenvolvimento dos sistemas orgânicos em estágios diferentes, relacionados à idade gestacional ${ }^{21}$.

Somente três pesquisas com metodologia semelhante à empregada neste estudo foram encontradas, pois o procedimento de fisioterapia neonatal empregado varia muito regionalmente e principalmente entre países, impossibilitando a comparação entre procedimentos diferentes. Em duas pesquisas realizadas, $\mathrm{Abreu}^{11,12}$ encontrou diminuição da FC e FR em RNPT após o procedimento de fisioterapia em concordância com este estudo, afirmando que os procedimentos de fisioterapia possuem subsídios teóricos e evidências práticas que corroboram para a maturação do sistema nervoso, gerando respostas motoras menos estereotipadas a cada sessão clínica de fisioterapia.

E em outra pesquisa Nicolau ${ }^{10}$ estudou variação dos parâmetros fisiológicos de 42 RNPT, em 5 momentos durante o PIFN e concluiu, em concordância com o nosso estudo, que os procedimentos de fisioterapia realizados não comprometeram a estabilidade clínica dos RNPT. Já Abreu et $\mathrm{a}^{25}$ estudaram a variabilidade da FC em RNPT com hemorragia periventricular-intraventricular e relataram que nos PIFN, quando se compara os pré-PIFN com os pós-PIFN, há um declíneo da FC de maneira linear. O mesmo comportamento dessa variável pode ser observado noutro estudo ${ }^{3}$ do ano de 2006 do mesmo grupo com população semelhante, entretanto com diagnóstico de doença pulmonar das membranas hialinas. Esses dados são concordantes com aqueles encontrados nesta pesquisa, ao se comparar o comportamento da variável fisio-metabólica da FC que também mantém a tendência linear de queda após os PIFN.

FOX et al ${ }^{13}$ estudaram 13 recém-nascidos para determinar a relação benefício e risco da fisioterapia respiratória. Todos estavam intubados e respirando espontaneamente com pressão posi- tiva em vias aéreas e se recuperando de enfermidades respiratórias. Aplicou-se, durante 30 segundos, vibração torácica com vibrador mecânico. Os recém-nascidos foram aspirados e hiperventilados por dez vezes. Houve uma tendência uniforme para aumento da complacência e de capacidade residual pulmonar. Observou-se que a resistência das vias aéreas caía significantemente após a vibração, porém, após duas horas, houve retorno aos níveis anteriores.

Os resultados obtidos evidenciaram a melhora clínica dos RNPT tratados, concordando com os resultados descritos por FOX et al ${ }^{13}$, ao demonstrar que as sessões clínicas de fisioterapia neonatal colaboraram para a redução das frequiências cardíaca e respiratória e aumento da saturação de oxigênio.

Existem relatos na literatura de que várias alterações na FC têm sido encontradas em resposta a intervenções ambientais, como o uso de consolabilidade e soluções orais de glicose. Gradin ${ }^{22}$ encontrou um aumento na FC em RNs que receberam glicose. Em nosso serviço utiliza-se a consolabilidade durante o procedimento de fisioterapia, porém não são utilizadas soluções orais de glicose.

Outra questão relacionada a fatores ambientais foi relatada num estudo realizado por Fernandez et $\mathrm{al}^{9}$ que estudaram $98 \mathrm{RNs}$ e concluíram que a luz e a temperatura ambiental são fatores que influenciam no ritmo circadiano e ultradiano da FR, variando em períodos de 3 horas, funcionando como fatores sincronizadores nas UCIN. Com base nesses dados, foi recomendado que as interpretações de variações dos parâmetros fisiológicos devam levar em consideração o horário das análises; por este motivo a presente pesquisa foi realizada sempre no mesmo horário.

Goto et $\mathrm{al}^{23}$ avaliaram a variabiliadade de FC em posição prona e supina em RNPT, e observaram que em posição prona a FC se mantém mais estável, o que explica o fato de termos mantido todos os RNs na mesma posição durante a realização do protocolo, evitando interferência do posicionamento sobre o resultado.

A temperatura corporal é o resultado do balanço entre termogênese e termólise na manutenção da homeostasia. Porém sabe-se que o RN é extremamente susceptível às alterações térmi- 
cas, sendo estas alterações mais acentuadas quanto menor for a idade gestacional e o peso ao nascimento ${ }^{24-26}$.

A condição mais favorável ao RN parece estar relacionada a um ambiente de termoneutralidade, considerada como a faixa de temperatura dentro da qual a taxa metabólica do RN é mínima e a temperatura corporal é mantida dentro da normalidade através do controle vasomotor e postural. A perda de calor do RNPT com idade gestacional abaixo de 30 semanas está aumentada devido à baixa capacidade de isolamento da pele, causada pela diminuição da queratinização do tecido subcutâneo, que propiciam maior perda de calor por evaporação. Além disso, os RNPT apresentam capacidade reduzida de vasoconstrição, por imaturidade do sistema vasomotor e uma grande superfície corpórea quando comparada ao seu peso ${ }^{26}$. Du- rante a realização do PIFN a incubadora permaneceu aberta, o que pode explicar a queda de temperatura encontrada neste estudo.

A queda de temperatura encontrada, por estar dentro dos valores de normalidade, foi considerada sem significância clínica.

\section{CONCLUSÃO}

A fisioterapia neonatal demonstrou ser um procedimento terapêutico sem repercussões deletérias em relação às variáveis fisio-metabólicas para o tratamento da população estudada.

Agradecimento: Aos profissionais da Unidade de Cuidados Intensivos Neonatal do Hospital Municipal Universitário de São Bernardo do Campo na colaboração para a coleta de dados

\begin{abstract}
The physiotherapy in the Neonatal Intensive Care Unit can be considerated a new kind of therapy. It's believed that the neonatal physiotherapeutic care is able to provide a stable heart and respiratory rate, blood pressure, and oxygen saturation, as well as maintain the corporal temperature, providing the functional maintenance of brain circulation in newborns and also of air ways with minimal turbulent flow and without lung secretion, allowing the increase on permeability and the decrease of air ways intrinsically risk factors that contribute to increase the lung resistance and decrease in physiological events of gases exchange. Other authors show injurious effects of physiotherapy regarding the neonatal population. This controversy about the neonatal respiratory physiotherapy induced to study the effects of such procedure in physiologic parameters. The objective was this paper considers the effects of neonatal physiotherapy practice on the heart and respiratory rate, arterial pressure, oxygen saturation and temperature in preterm newborn babies submitted to mechanical ventilation. A prospective study about the neonatal population of the Hospital Municipal Universitário de São Bernardo do Campo, was carried out from January to December of 2005. A descriptive analysis of data was performed for the results. The Anova test, a model of variance analysis to repetitive measure, was done to compare if average values before and after the physiotherapy are statistically significant. A Newman-Keuls test was performed right away to heart and respiratory rate and oxygen saturation, and the T-test to arterial pressure and temperature. The significance level considered was $5 \%$. The sample consisted of 27 preterm newborns, treated with invasive mechanical ventilation and interventionist procedures of physiotherapy. A reduction of heart and respiratory rate along the clinical sessions of neonatal physiotherapy was observed, with maintenance of the arterial pressure, increase of the $\mathrm{SpO} 2$ and reduction of the temperature. However, there have been no clinical repercussions in this population after interventionist procedure of physiotherapy. Many factors can influence on stability of physiological parameters, as well as monitoring each one of them, such as the neonatal physiology characteristics, enviroment factors and the type of intervention. In other words, the physiotherapy that is adjusted to each newborn situation, will determine the possible results regarding physiologic parameters in preterm newborns. In conclusion, the neonatal physiotherapy was shown to be a suitable therapeutical procedure for the treatment of preterm newborns.
\end{abstract}

Key words: Hemodynamic stability. Neonatal physiotherapy. Physiological parameters. Newborn. 


\section{REFERÊNCIAS}

1. Abreu LC, Giancio CD, Falcão MC, Oliveira AG, Saldiva PHN. Procedimentos fisioterapêuticos na síndrome de aspiração meconial. Rev. Fisioterapia do UniFMU 2003; 2:19-27.

2. Miyoshi MH, Guinsburg R, Kopelman BI, Kantorowitz J, Rodrigues SA. Terapêutica de reposição com surfactante exógeno em recémnascidos prematuros com Síndrome do Desconforto Respiratório. J. pediatr. 1993; 69(4):235-43.

3. Abreu LC, Angheben JMM, Braz PF, Oliveira AG, Falcão MC, Saldiva PHN. Effect of the neonatal physiotherapy in the heart rate in preterm infant with respiratory distress syndrome after replacement of exogenous surfactant. Arq Med ABC 2006; 31(1):5-11.

4. Oberwaldner B. Physiotherapy for airway clearance in pediatrics. Eur Respir J 2000; 15(1):196-204.

5. Avery ME, Mead J. Surface properties in relation to atelectasis and hyaline membrane disease. Am. J. Dis. Child. 1959; 97:517-23.

6. Flanady VJ, Gray BH. Chest Physiotherapy for preventing morbidity in babies being extubated from mechanical ventilation. The Cochrane Library (banco de dados), 4th ed. 2000.

7. Fernandez JA, Llano JMA, Gomez JA, Ramos MAR, Casado MLB, Suarez JG. Ritmo Biológico de la frequiência respiratória em el primer trimestre de la vida. An. Esp. Pediatr. 1992, 37(5): 377-382.

8. Antunes LCO, Silva EG, Bocardo P, Daher DR, Faggiotto RD, Rugolo LMSS. Efeitos da fisioterapia respiratória convencional versus aumento de fluxo expiratório na saturação de oxigênio, freqüência cardíaca e freqüência respiratória em prematuros no período pós extubação. Revista Bras Fisioter, 2006, 10:97103.

9. Abreu, LC. Impacto da fisioterapia neonatal em recém-nascidos pré-termo com doença pulmonar das membranas hialinas em ventilação mecânica e pós-reposição de surfactante exógeno. In: tese de Doutorado Universidade Federal de São Paulo - São Paulo, 2003.

10. Nicolau, CM. Repercussões da fisioterapia respiratória sobre a função cardiopulmonar em RNPT submetidos à ventilação mecânica. In: dissertação de mestrado - Universidade Federal de São Paulo - São Paulo, 2006.

11. Ropper PC, Vonwiller JB, Fisk GC, Gupta JM. Lobar atelectasys after nasotracheal intubation in newborn infants. Aust. Pediatr. J. 1976, 12:272-275.

12. Finner NN, Boyd J. Chest physitherapy in the neonate. A controled stydy. Pediatrics, 1978, 61:282-285.

13. Fox WW, Schwants JC, Shaffer TH. Alterations in neonatal respiratory function follonwing chest physiotherapy (abstract 1192). Pediatr. Res. 1977,11: 570.

14. Tozi EB, Dotta ML. Fisioterapia. In: Terapia Intensiva Pediátrica. $2^{a}$ ed., São Paulo: Atheneu; 1997. p. 1154-60.

15. Janoski JCB. Abordagem da fisioterapia repiratória em paciente pediátrico. In: Fisioterapia em Movimento, 1998.

16. Cheng M, Williams PD. Oxygenation during chest physiotherapy of very-low-birth-weight infants: relations among fraction of inspired oxygen levels, number of hand ventilations and transcutaneous oxygen pressure. Journal of Pediatric Nursing, 1989, 4(6): 411-8.

17. Harding JE, Miles FK, Becroft DM, Allen BC, Knight DB. Chest physiotherapy may be associated with brain damage in extremely premature infants. J Pediatr, 1998, 132(3):440-4.

18. Krause MF, Hoehn T. Effizienz und Risiken der Atemphysiotherapie beim NeugeborenenUbersicht uber die Literatur. / Efficiency and risk of chest physiotherapy in the newbornreview of the literature. Klin Padiatr, 1999, 211(1): 11-7.

19. Silva EG, Antunes LCO, Cabrera SF, Rugolo L. Efetividade e segurança da fisioterapia respiratória convencional versus aumento de fluxo expiratório em prematuros sob ventilação mecânica. Revista Bras Fisioter. 2006, Supl.09:18.

20. Capurro H, Konichezky S, Fonseca D, Caldeyro-barcia R. A Simplified method for diagnostic of gestacional age in the newborn infant. J. Pediatr. 1978, 93:102-12.

21. Hack M, Horbar JD, Malloy M, Tyson JE, Wright E, Wright L. Very low weight outcomes of National Institute of Child Health and Human Development Neonatal Network. Pediatrics. 1991, 1987:587-97.

22. Gradin M. Effect of oral glucose on the hearth rate of healthy newborns. Acta Paediatr. 2005, 94(3): 324-8. 
23. Goto K, Maeda T, Mirmiram M; Ariagno R. Effects of prone and supine position on sleep characteristics in preterm infants. Psychiatry Clin Neurosci. 1999, 53(2):315-17.

24. Abreu LC. Efeitos terapêuticos da fisioterapia pulmonar e motora em recém-nascidos prétermo com hemorragia periventricularintraventricular. In: tese de Mestrado Universidade Federal de São Paulo - São Paulo, 1998.
25. Abreu LC, Falcão MC, Oliveira AG, Saldiva PHN. Efeitos terapêuticos da fisioterapia neonatal sobre a freqüência cardíaca em recém-nascido pré-termo com hemorragia periventricularintraventricular. Fisioterapia Brasil, Rio de Janeiro, RJ - Brasil, v. 4, n. 12, p. 19-27, 2003.

26. Nascimento SD. Termorregulação no recémnascido. In: Diagnóstico e tratamento em neonatologia. Atheneu, São Paulo, 2004.

Recebido em 07/11/2006 Modificado em 22/11/2006 Aprovado em 30/11/2006 